\title{
Analysis of cool DO-type white dwarfs from the Sloan Digital Sky Survey data release 10
}

\author{
N. Reindl ${ }^{1}$, T. Rauch ${ }^{1}$, K. Werner ${ }^{1}$, S. O. Kepler ${ }^{2}$, B. T. Gänsicke ${ }^{3}$, and N. P. Gentile Fusillo ${ }^{3}$ \\ ${ }^{1}$ Institute for Astronomy and Astrophysics, Kepler Center for Astro and Particle Physics, Eberhard Karls University, Sand 1, \\ 72076 Tübingen, Germany \\ e-mail: reindl@astro.uni-tuebingen.de \\ 2 Instituto de Física, Universidade Federal do Rio Grande do Sul, 91501-900 Porto Alegre, RS, Brazil \\ 3 Department of Physics, University of Warwick, Coventry, CV4 7AL, UK
}

Received 26 August 2014 / Accepted 17 October 2014

\begin{abstract}
We report on the identification of 22 new cool DO-type white dwarfs (WDs) detected in data release 10 (DR10) of the Sloan Digital Sky Survey (SDSS). Among them, we found one more member of the so-called hot-wind DO WDs, which show ultrahigh excitation absorption lines. Our non-LTE model atmosphere analyses of these objects and two not previously analyzed hot-wind DO WDs, revealed effective temperatures and gravities in the ranges $T_{\text {eff }}=45-80 \mathrm{kK}$ and $\log g=7.50-8.75$. In eight of the spectra we found traces of $C$ (0.001-0.01, by mass). Two of these are the coolest DO WDs ever discovered that still show a considerable amount of $C$ in their atmospheres. This is in strong contradiction with diffusion calculations, and probably, similar to what is proposed for DB WDs, a weak mass-loss is present in DO WDs. One object is the most massive DO WD discovered so far with a mass of $1.07 M_{\odot}$ if it is an $\mathrm{ONe}-\mathrm{WD}$ or $1.09 M_{\odot}$ if it is a CO-WD. We furthermore present the mass distribution of all known hot non-DA (pre-) WDs and derive the hot DA to non-DA ratio for the SDSS DR7 spectroscopic sample. The mass distribution of DO WDs beyond the wind limit strongly deviates from the mass distribution of the objects before the wind limit. We address this phenomenon by applying different evolutionary input channels. We argue that the DO WD channel may be fed by about $13 \%$ by post-extreme-horizontal branch stars and that PG 1159 stars and $\mathrm{O}(\mathrm{He})$ stars may contribute in a similar extent to the non-DA WD channel.
\end{abstract}

Key words. stars: abundances - stars: evolution - stars: AGB and post-AGB - white dwarfs

\section{Introduction}

The vast majority of stars is expected to end as a white dwarf (WD), most of them $(\approx 80 \%)$ with H-rich atmospheres, corresponding to the DA spectral type. These can be found all along the WD cooling sequence, that is, they have $4500 \leq T_{\text {eff }} \leq$ $170000 \mathrm{~K}$ (Sion 2011). In addition, there are the H-deficient WDs (non-DA WDs), which are usually divided into three subclasses: the DO spectral type $\left(45000 \leq T_{\text {eff }} \leq 200000 \mathrm{~K}\right)$, with the hot DO WDs showing strong He II lines, whereas in the spectra of cool DO WDs He I lines can also be seen; the DB type $\left(11000 \leq T_{\text {eff }} \leq 45000 \mathrm{~K}\right)$ showing strong He I lines; and the DC (featureless spectra), DQ, and DZ types ( $\left.T_{\text {eff }} \leq 11000 \mathrm{~K}\right)$ showing traces of carbon and other metals in their spectra (Sion 2011). The overlap in temperature of the hottest DA and nonDA WDs strongly suggests the existence of a separate evolutionary channel for both classes. While a direct evolutionary connection for the H-rich central stars of planetary nebulae to the DA WDs is very likely (Napiwotzki \& Schönberner 1995), the formation and evolution of non-DA WDs is less well understood.

DO WDs are commonly believed to be the successors of the PG 1159 stars (e.g., Werner et al. 2014; Althaus et al. 2009), which are hot $\left(75000 \leq T_{\text {eff }} \leq 200000 \mathrm{~K}\right)$ stars that show $\mathrm{H}$-deficient and He-, C-, and O-rich surface compositions (typically He: $\mathrm{C}: \mathrm{O}=0.30-0.85: 0.15-0.60: 0.02-0.20$ by mass, Werner \& Herwig 2006). These abundances can be explained by a very late thermal pulse (VLTP), experienced by a WD during its early cooling phase (Iben et al. 1983; Althaus et al. 2005b). Most of the residual hydrogen envelope is engulfed by the helium-flash convection zone and completely burned at the beginning of this thermal pulse. The star is then forced to rapidly evolve back to the AGB and finally into a hydrogen-deficient, helium-burning PG 1159 star (Althaus et al. 2005b). As the star cools down, gravitational settling removes heavy elements from the photosphere and turns it into a DO WD (Unglaub \& Bues 2000 , unless it is of the subtype hybrid-PG 1159, then it turns into a DA WD).

In the past years it became more clear that the non-DA WDs are fed by distinct $\mathrm{H}$-deficient evolutionary channels. In addition to the carbon-dominated sequence, a helium-dominated sequence exists (Althaus et al. 2009; Miszalski et al. 2012; Reindl et al. 2014; Frew et al. 2014). VLTP scenarios fail to reproduce the helium-rich atmospheres ( $\mathrm{He} \geq 95 \%$, by mass) of He-rich subdwarf $\mathrm{O}$ (sdO) stars, R Coronae Borealis (RCB) stars, extreme helium (EHe) stars, [WN]-type central stars, and $\mathrm{O}(\mathrm{He})$ stars, suggesting that these objects have a different formation history. The origin of these stars remains uncertain. Their abundances match predictions of a double-helium WD merger scenario (Zhang \& Jeffery 2012a,b), suggesting the evolutionary channel $\mathrm{sdO}(\mathrm{He}) \rightarrow \mathrm{O}(\mathrm{He}) \rightarrow \mathrm{DO}$ WD or, in case of Cand $\mathrm{N}$-rich sdO and $\mathrm{O}(\mathrm{He})$ stars, $\mathrm{RCB} \rightarrow \mathrm{EHe} \rightarrow \mathrm{sdO}(\mathrm{He}) \rightarrow$ $\mathrm{O}(\mathrm{He}) \rightarrow$ DO WD (Reindl et al. 2014). The existence of planetary nebulae that do not show helium enrichment around every other $\mathrm{O}(\mathrm{He})$ star or [WN]-type central star, however, precludes a double-helium WD merger origin for these stars. These stars must have formed in a different way, for instance, by enhanced mass-loss during their post-AGB evolution, or a merger within a common envelope of a CO-WD and a red giant or AGB star (Reindl et al. 2014). 
Dufour et al. (2007) reported the discovery of several WDs with atmospheres primarily composed of carbon, with little or no trace of hydrogen or helium. These stars do not fit satisfactorily in any of the currently known theories of post-AGB evolution. They are considered to be the cooler counterpart of the unique and extensively studied PG1159 star H1504+65 (Nousek et al. 1986; Werner 1991; Werner et al. 2004a) and might form a new evolutionary sequence that follows the AGB. Another case of $\mathrm{H}$-deficient WDs that need to fit into the evolutionary picture are the oxygen-dominated WDs discovered by Gänsicke et al. (2010).

As a result of their fast evolutionary rate, there are only a few very hot $\mathrm{H}$-deficient stars. A literature study revealed that there are currently only 46 known PG 1159 stars, $10 \mathrm{O}(\mathrm{He})$ stars, and 52 DO WDs ${ }^{1,2}$. The detection and analysis of new DO WDs improve their statistics and thus helps to understand the origin of those objects. Furthermore, it is of importance for the construction of the hot end of the WD luminosity function. Its shape is an excellent tool for constraining the emission of particles in the core of hot DO WDs, for example, and to check for the possible existence of DFSZ axions, a proposed but not yet detected type of weakly interacting particles (Miller Bertolami et al. 2014; Miller Bertolami 2014).

In this paper, we first describe the observations and line identifications (Sect. 2). The spectral analysis follows in Sect. 3. The results are discussed in Sect. 4, where we mention the phenomenon of the so-called hot-wind DO WDs (Sect. 4.1) and debate the evolution of the $\mathrm{C}$ abundances before and along the nonDA WD cooling sequence (Sect. 4.2). The mass distribution of all known $\mathrm{O}(\mathrm{He})$ stars, PG 1159 stars, and DO WDs is presented in Sect. 4.3 and the hot DA to non-DA ratio in Sect. 4.4.

In the text, we use abbreviated versions of the object names. Full names are given in the Tables 1 and 2.

\section{Observations and line identifications}

Until 1996, only 22 DO WDs $^{3}$ were known (Dreizler \& Werner 1996). Hügelmeyer et al. $(2005,2006)$ almost doubled this number with objects from the SDSS DR1, DR2, DR3, and DR4. Recently, Werner et al. (2014) detected ten new hot DO WDs in the SDSS DR 10. To complete the sample of DO WDs, we have visually scanned a color-selected sample of WD candidates in the SDSS DR10 and also searched for cool DO WDs. These are distinguished from the hot WDs by the presence of He I lines. To distinguish cool DO WDs from sdO stars, we only considered spectra with broad and only weak higher order $(n \geq 10)$ He II lines. We found 24 objects (Figs. 1 and 2) that have not previously been analyzed with non-local thermodynamic equilibrium (LTE) model atmospheres. This sample includes two previously not analyzed hot-wind DO WDs (see below) and almost doubles the number of cool DO WDs below $80 \mathrm{kK}$.

Of the 24 stars, 14 were already included in the DR7 and also found by Kleinman et al. (2013). These authors classified 11 of the stars as DO WDs, two as DOBAH (J1442, J1509) and one as DBAH (J0005). The latter was already included in the SDSS DR4 catalog of spectroscopically confirmed WDs (Eisenstein et al. 2006) and classified as an sdO star. The analyses of

\footnotetext{
This excludes the 22 new DO WDs from this paper.

2 A list of all analyzed DO WDs can be found at http://astro. uni-tuebingen.de/ reindl/He

3 This excludes KPD 0005+5106 which was recently reclassified as a pre-WD (Werner et al. 2014) and is now considered as an $\mathrm{O}(\mathrm{He})$ star (Reindl et al. 2014).
}

Table 1. DO WD candidates from the SDSS DR7 WD catalog (Kleinman et al. 2013) that were rejected from our sample.

\begin{tabular}{lcrl}
\hline \hline SDSS name & $g[\mathrm{mag}]$ & $S / N$ & Comment \\
\hline J012602.53-004834.5 & 17.97 & 20 & misclassified He-sdO \\
J014531.86+010629.7 & 20.15 & 8 & poor quality spectrum \\
J024323.23+275045.5 & 19.48 & 12 & poor quality spectrum \\
J025622.18+330944.7 & 19.70 & 10 & poor quality spectrum \\
J065745.83+834958.5 & 19.18 & 18 & poor quality spectrum \\
J080846.19+313106.0 & 19.44 & 53 & misclassified CV \\
J081533.08+264646.4 & 19.48 & 19 & poor quality spectrum \\
J083959.93+142858.0 & 18.61 & 6 & poor quality spectrum \\
J094526.91+172917.2 & 20.28 & 5 & poor quality spectrum \\
J102907.31+254008.4 & 17.35 & 16 & misclassified DAO \\
J130249.00-013309.5 & 19.15 & 9 & poor quality spectrum \\
J130249.00-013309.5 & 18.71 & 11 & poor quality spectrum \\
J131816.55+485741.3 & 19.15 & 7 & poor quality spectrum \\
J151246.56+071517.3 & 19.41 & 13 & poor quality spectrum \\
J154829.87+203139.1 & 16.81 & 35 & misclassified DAO \\
J155642.95+501537.5 & 15.81 & 43 & misclassified He-sdO \\
J161512.22+110240.0 & 16.89 & 36 & misclassified DAO \\
J163200.32-001928.3 & 18.37 & 20 & misclassified DAO \\
J171600.52+422131.1 & 18.19 & 16 & poor quality spectrum \\
J173027.20+265639.5 & 17.07 & 33 & misclassified He-sdO \\
J173824.64+581801.8 & 18.36 & 13 & poor quality spectrum \\
J205030.40-061957.9 & 17.98 & 21 & misclassified DAO \\
J205930.25-052848.9 & 17.64 & 25 & misclassified DAO \\
J213932.49+112611.3 & 19.29 & 9 & poor quality spectrum \\
\hline
\end{tabular}

Kleinman et al. (2013) and Eisenstein et al. (2006) were only based on LTE models and, thus do not provide reliable atmospheric parameters for DO WDs. Kleinman et al. (2013) listed 52 additional DO WDs, 28 of them previously analyzed with non-LTE model atmospheres. The remaining 24 had spectra with a too low signal-to noise ratio $(\mathrm{S} / \mathrm{N})$ or were either a misclassified cataclysmic variable $(\mathrm{CV})$, misclassified He-sdO stars $(\log g \leq 7.0)$, or DAO WDs. J0839 is listed as DAO WD, but might be a good candidate for a DO WD. The poor S/N, however, does not allow a precise spectral analysis. All these objects are listed in Table 1 and were rejected from our sample.

Table 2 lists all the DO WDs from our sample. In the spectra of six newly discovered objects (J0742, J0902, $\mathrm{J} 1107, \mathrm{~J} 1531, \mathrm{~J} 1707$, and J1717), we were able to detect the $\mathrm{C}_{\text {IV }} \lambda \lambda 4647,4657,4658,4659,4660,5803,5814 \AA$ lines. For the first time, we identified C III lines in the spectra of two DO WDs. In the spectrum of J2239, we found $\mathrm{C}_{\text {III }} \lambda \lambda 4515,4516,4517,4647$, and $4650 \AA$, and in the spectrum of J0301, we additionally identified C III $\lambda \lambda$ 4056, 4068, 4069, 4070, 4187, and 4326.

Our sample furthermore includes three members of the socalled hot-wind DO WDs, which show ultrahigh excitation (uhei) absorption lines. The uhei lines in the spectrum of J0747 (HS 0742+6520) were identifed for the first time. This star was first classified as an $\mathrm{O}(\mathrm{He})$ star by Heber et al. (1996), who discovered it in the Hamburg-Schmidt survey. However, because of the low $\mathrm{S} / \mathrm{N}$, the uhei features were not clearly visible in this spectrum. Sinamyan (2011) used empirical formulas to estimate $T_{\text {eff }}$ and $\log g$ for 87 First Byurakan Survey (FBS) WDs. Based on the SDSS colors, he found for J0747 $T_{\text {eff }}=85279 \mathrm{~K}$ and $\log g=7.78$. J0201 (HS 0158+2335, discovered by Dreizler et al. 1995) and J0717 (also known as HS 0713+3958, discovered by Werner et al. 1995) are known DO WDs with uhei features. Since their SDSS spectra range up to $10000 \AA$, we were able to identify additional lines beyond the hitherto observed $\mathrm{H} \alpha$ region (Table 3). Figure 2 shows the spectra of these DO WDs. 
Table 2. Parameters of the new cool DO WDs.

\begin{tabular}{|c|c|c|c|c|c|c|}
\hline SDSS name & $\begin{array}{l}T_{\text {eff }} \\
{[\mathrm{kK}]}\end{array}$ & $\begin{array}{l}\log g \\
{\left[\mathrm{~cm} / \mathrm{s}^{2}\right]}\end{array}$ & $C$ & $\begin{array}{l}M \\
{\left[M_{\odot}\right]}\end{array}$ & $\begin{array}{l}g \\
{[\mathrm{mag}]}\end{array}$ & Remarks \\
\hline J000509.93+003809.6 & $51_{-2}^{+2}$ & $8.00 \pm 0.25$ & $<0.003$ & $0.64_{-0.10}^{+0.14}$ & 18.181 & \\
\hline J020127.20+234952.7 & $70_{-10}^{+10}$ & $7.75 \pm 0.50$ & $<0.002$ & $0.58_{-0.06}^{+0.11}$ & 16.633 & HS $0158+2335$, uhei \\
\hline J030130.10+050802.9 & $50_{-1}^{+2}$ & $7.75 \pm 0.25$ & $0.01_{-0.005}^{+0.01}$ & $0.54_{-0.07}^{+0.10}$ & 18.392 & \\
\hline J071644.59+395808.9 & $60_{-5}^{+10}$ & $8.25 \pm 0.50$ & $<0.05$ & $0.75_{-0.09}^{+0.15}$ & 18.591 & \\
\hline J071702.72+395323.6 & $80_{-10}^{+10}$ & $7.75 \pm 0.50$ & $<0.003$ & $0.60_{-0.07}^{+0.09}$ & 16.295 & HS 0713+3958, uhei \\
\hline J072025.52+370336.3 & $52_{-2}^{+2}$ & $7.75 \pm 0.25$ & $<0.01$ & $0.54_{-0.07}^{+0.10}$ & 18.271 & \\
\hline J073849.46+485126.6 & $60_{-5}^{+10}$ & $8.00 \pm 0.50$ & $<0.01$ & $0.66_{-0.16}^{+0.26}$ & 18.695 & \\
\hline J074203.59+493333.8 & $60_{-5}^{+10}$ & $7.75 \pm 0.25$ & $0.002_{-0.001}^{+0.001}$ & $0.56_{-0.06}^{+0.10}$ & 16.707 & \\
\hline J074725.15+651301.1 & $80_{-10}^{+10}$ & $7.50 \pm 0.50$ & $<0.002$ & $0.53_{-0.08}^{+0.16}$ & 15.414 & HS $0742+6520$, uhei \\
\hline J075633.77+163359.0 & $50_{-2}^{+2}$ & $7.50 \pm 0.25$ & $<0.005$ & $0.49_{-0.04}^{+0.05}$ & 18.891 & \\
\hline J081739.99+163117.1 & $60_{-9}^{+10}$ & $7.50 \pm 0.50$ & $<0.05$ & $0.50_{-0.04}^{+0.06}$ & 19.184 & \\
\hline $\mathrm{J} 090227.65+125206.0$ & $60_{-5}^{+5}$ & $7.75 \pm 0.25$ & $0.003_{-0.001}^{+0.002}$ & $0.56_{-0.06}^{+0.10}$ & 17.049 & \\
\hline J090958.98+011105.4 & $51_{-2}^{+2}$ & $7.50 \pm 0.25$ & $<0.003$ & $0.49_{-0.04}^{+0.05}$ & 16.660 & \\
\hline $\mathrm{J} 103041.44+401312.6$ & $55_{-3}^{+5}$ & $8.00 \pm 0.25$ & $<0.005$ & $0.65_{-0.16}^{+0.13}$ & 17.348 & \\
\hline $\mathrm{J} 110747.86+383550.8$ & $60_{-5}^{+5}$ & $8.00 \pm 0.25$ & $0.003_{-0.001}^{+0.002}$ & $0.66_{-0.10}^{+0.12}$ & 17.173 & \\
\hline J113816.30+382635.1 & $65_{-10}^{+10}$ & $8.75 \pm 0.50$ & $<0.01$ & $1.08_{-0.29}^{+0.18}$ & 18.080 & \\
\hline $\mathrm{J} 130717.27+004151.6$ & $60_{-10}^{+10}$ & $8.00 \pm 0.50$ & $<0.01$ & $0.66_{-0.16}^{+0.26}$ & 17.188 & \\
\hline $\mathrm{J} 142440.79+330439.5$ & $50_{-2}^{+2}$ & $7.50 \pm 0.25$ & $<0.005$ & $0.49_{-0.04}^{+0.05}$ & 18.572 & \\
\hline J150927.22+292206.3 & $50_{-1}^{+2}$ & $7.75 \pm 0.25$ & $<0.003$ & $0.54_{-0.07}^{+0.10}$ & 17.321 & \\
\hline $\mathrm{J} 152943.01+183722.8$ & $52_{-3}^{+3}$ & $7.50 \pm 0.25$ & $<0.005$ & $0.49_{-0.04}^{+0.05}$ & 18.432 & \\
\hline $\mathrm{J} 153133.11+343327.5$ & $70_{-5}^{+10}$ & $8.00 \pm 0.25$ & $0.003_{-0.001}^{+0.002}$ & $0.69_{-0.11}^{+0.09}$ & 15.834 & \\
\hline $\mathrm{J} 170723.85+450009.9$ & $60_{-5}^{+10}$ & $8.00 \pm 0.50$ & $0.003_{-0.001}^{+0.002}$ & $0.66_{-0.16}^{+0.26}$ & 18.644 & \\
\hline $\mathrm{J} 171720.52+373605.9$ & $60_{-5}^{+5}$ & $7.75 \pm 0.25$ & $0.003_{-0.001}^{+0.002}$ & $0.56_{-0.06}^{+0.10}$ & 17.880 & \\
\hline J223939.44+225925.9 & $45_{-2}^{+1}$ & $7.50 \pm 0.25$ & $0.001_{-0.0009}^{+0.002}$ & $0.47_{-0.05}^{+0.05}$ & 18.248 & \\
\hline
\end{tabular}

Notes. C abundances are given as mass fraction. uhei indicates objects with ultrahigh excitation features.

\section{Spectral analysis}

We used the Tübingen non-LTE model-atmosphere package (TMAP ${ }^{4}$, Werner et al. 2003; Rauch \& Deetjen 2003) to compute non-LTE, plane-parallel, fully metal-line-blanketed model atmospheres in radiative and hydrostatic equilibrium. The model atoms for this analysis were taken from the Tübingen modelatom database $\mathrm{TMAD}^{5}$. To calculate synthetic line profiles, we used Stark line-broadening tables provided by Barnard et al. (1969) for He I $\lambda \lambda 4026,4388,4471,4921 \AA$, Barnard et al. (1974) for He I $\lambda 4471 \AA$ and Griem (1974) for all other He I lines, and for He II and C IV we used the tables provided by Schöning \& Butler (1989) and Schöning (1993). To account for the spectral resolution of the observations, synthetic spectra were convolved with Gaussians ( $F W H M=2.5 \AA)$. All observed spectra were shifted to rest wavelengths by applying radialvelocity corrections by centroiding the He I and He II lines.

First, we calculated a pure-He model grid, spanning from $T_{\text {eff }}=45-90 \mathrm{kK}$ (in steps of $5 \mathrm{kK}$ ) and $\log g=6.75-8.75$ (in steps

\footnotetext{
4 http://astro.uni-tuebingen.de/ TMAP

5 http://astro.uni-tuebingen.de/ TMAD
}

of 0.25$)$. Because the $\mathrm{He}$ / $/ \mathrm{He}$ II ionization equilibrium is very sensitive around $T_{\text {eff }}=50 \mathrm{kK}$, we refined the model grid from $T_{\text {eff }}=43-55 \mathrm{kK}$ to $1 \mathrm{kK}$ steps. We reproduced all He lines and chose the best-fit models by visual comparison with the whole rectified observed WD spectra. To determine $T_{\text {eff }}$, we used the $\mathrm{He}$ I/ He II ionization equilibrium, and for the $\log g$ determination the wings of the He lines.

Then, we also included $\mathrm{C}(C=0.0001,0.0005, C=$ $0.001-0.01$, in 0.001 steps, and $C=0.01-0.05$, in 0.01 steps, by mass) into our best-fit models to derive $\mathrm{C}$ abundances by fitting all identified $\mathrm{C}$ lines (Sect. 2). Upper limits were derived by test models where the respective lines in the model contradicted the non-detection of the lines in the observation (at the abundance limit). For five stars (J0902, J1107, J1531, J1707, and J1717), we derived $C=0.003$, which is slightly supersolar (solar abundance according to Asplund et al. 2009). For J0742 we found $C=0.002$ (about solar). Two of our objects are the coolest DO WDs ever discovered that still show a considerable amount of C. J0301 ( $\left.T_{\mathrm{eff}}=50 \mathrm{kK}\right)$ was found to have the highest $\mathrm{C}$ abundance in our sample ( $C=0.01$, about four times solar), and for $\mathrm{J} 2239\left(T_{\mathrm{eff}}=45 \mathrm{kK}\right)$ we found $C=0.001$ (slightly subsolar). 


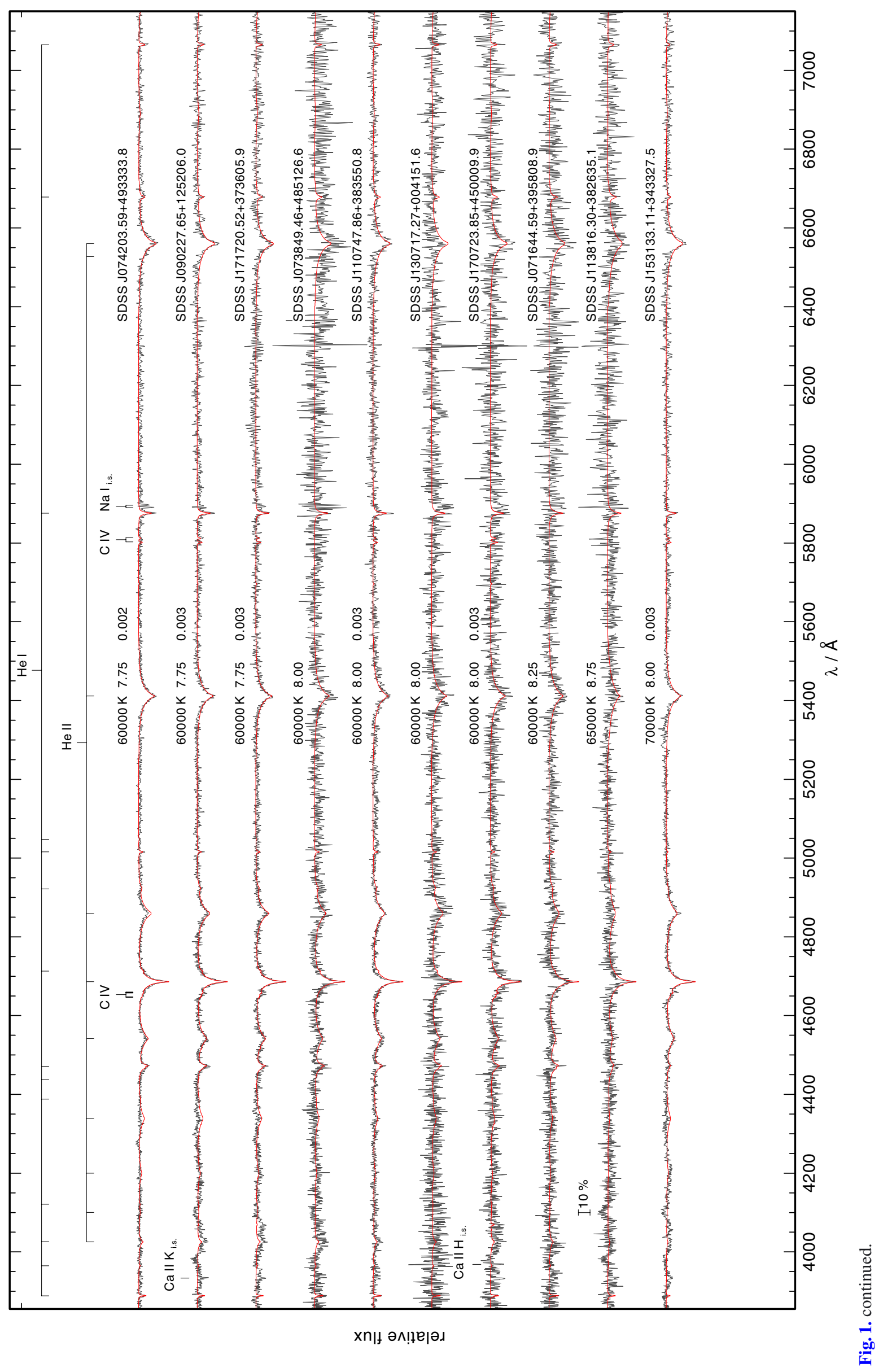

A117, page 5 of 12 
A\&A 572, A117 (2014)

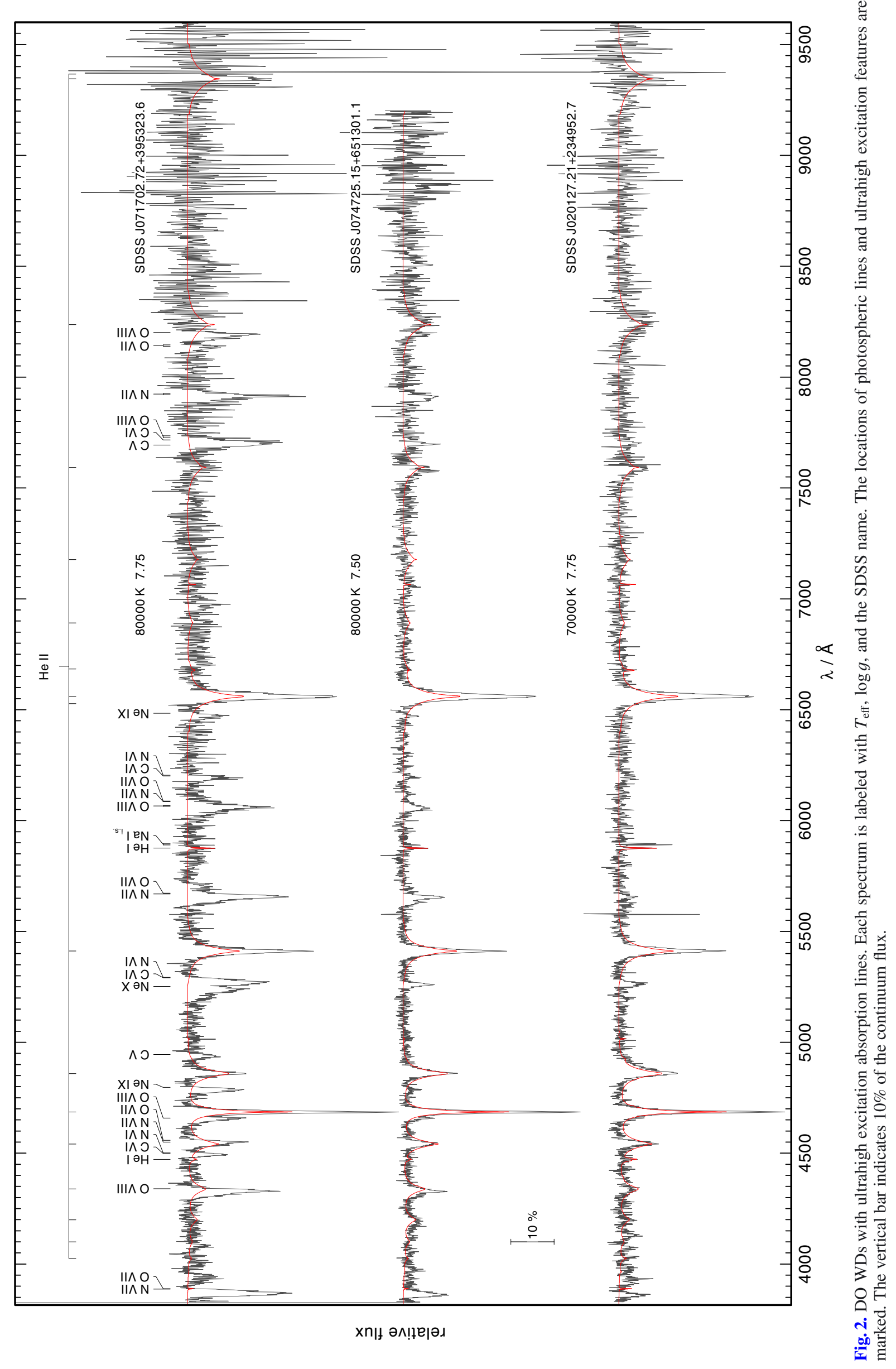


The three DO WDs with uhei features clearly show He I $\lambda 5875.7 \AA$; the high S/N of the spectra of J0747 and J0201 also allowed the identification of He I $\lambda 4471.5 \AA$. We used these lines to constrain $T_{\text {eff }}$ and found $T_{\text {eff }}=70 \mathrm{kK}$ for $\mathrm{J} 0201$ and $T_{\text {eff }}=80 \mathrm{kK}$ for J0717 and J0747. For the surface gravity of these objects we estimate $\log g=7.75$ (for J0717 and J0201) and $\log g=7.50$ (for J0747). For lower values of $\log g$, the Pickering lines below $4500 \AA$ become too strong. Upper limits for $\log g$ were derived using He II $\lambda \lambda 4542,4860,8237 \AA$, which form deep in the atmosphere and might thus be least affected by the process that creates the too-deep He II lines. We summarize the results of our analysis in Table 2.

The additional opacities of $\mathrm{C}$ do not affect the theoretical $\mathrm{He}$ I and He II line profiles. We also investigated a possible impact of line blanketing by iron-group elements. For that, we calculated two test models with $T_{\text {eff }}=60,80 \mathrm{kK}$, and $\log g=7.0$. Iron-group elements were included with a generic model atom (Rauch \& Deetjen 2003) containing the elements $\mathrm{Ca}, \mathrm{Sc}, \mathrm{Ti}, \mathrm{V}, \mathrm{Cr}, \mathrm{Mn}$, $\mathrm{Fe}, \mathrm{Co}$, and $\mathrm{Ni}$ at solar abundance values. For the $T_{\mathrm{eff}}=60 \mathrm{kK}$ model the ionization stages IV - VII were considered and for the $T_{\text {eff }}=80 \mathrm{kK}$ model the ionization stages $\mathrm{V}-$ IX. The model atom was calculated via the Tübingen iron-group opacity interface TIRO $^{6}$ (Müller-Ringat 2013). This has recently been developed in the framework of the Virtual Observatory $\left(\mathrm{VO}^{7}\right)$ and is provided as a registered service by the German Astrophysical Virtual Observatory ${ }^{8}$. We found that for the pure-He models, the central depression of He II $\lambda 4686 \AA$ is stronger than in the model that includes the iron-goup elements ( $42 \%$ stronger in the $T_{\text {eff }}=60 \mathrm{kK}$ model and $10 \%$ stronger in the $T_{\text {eff }}=80 \mathrm{kK}$ model $)$. However, the profiles of all the other He I and He II lines remain unaffected.

\section{Discussion}

\subsection{Hot-wind DO WDs}

The discovery of uhei lines and unusually deep He II lines in the spectrum of J0747 makes it the eleventh member of the socalled hot-wind DO WDs. This class so far included eight other DO WDs: SDSS J105956.00+404332.4 (Werner et al. 2014), SDSS J151026.48+610656.9, $\quad$ SDSS J025403.75+005854.4 (Hügelmeyer et al. 2006), HS 0158+2335, HS 0727+6003, HS 2027+0651 (Dreizler et al. 1995), HE 0504-2408, HS 0713+3958 (Werner et al. 1995), one PG 1159 star: SDSS J121523.09+120300.8 (Hügelmeyer et al. 2006), and one DAO WD HS 2115+1148 (Dreizler et al. 1995). In addition, there are five other DO WDs known that also show too deep He II lines that they cannot be fitted by any model, but have no clear uhei features in their spectra: SDSS J082134.95+173919.40, SDSS J082724.44+585851.68， SDSS J094722.49+101523.62, SDSS J151215.72+065156.34 (Werner et al. 2014), and HE 1314+0018 (Werner et al. 2004b; Hügelmeyer et al. 2006). Werner et al. (2014) speculated that the same unknown process is at work here, affecting the He II lines, but failing to generate the strong uhei lines.

An attempt was made by Werner et al. (1995) to explain the uhei absorptions lines by extremely hot, static, plane-parallel non-LTE model atmospheres. The results showed that the observed spectra cannot have a photospheric origin assuming such an extreme $T_{\text {eff }}$. Since the strongest of the uhei lines often show

\footnotetext{
6 http://astro.uni-tuebingen.de/ TIRO

http://www.ivoa.net

8 http://wwW.g-vo.org
}

a very broad blue wing, it is believed that they are formed in a hot stellar wind that is optically thick in these transitions along the line of sight toward the stellar disk.

From constraining atmospheric parameters for these stars with the method described in Sect. 3, we cannot find any correlation of the temperature (e.g., the presence of He I lines), and the presence or strength of uhei features in these DO WDs.

The fraction of DO WDs showing this phenomenon is significant (19\% show too deep He II lines, $11 \%$ show additional uhei features) and awaits an explanation.

\section{2. $C$ abundances}

The detection of $\mathrm{C}$ in cool DO WDs is of particular interest in studying the chemical evolution of WDs and to place constraints on possible progenitors and successors of DO WDs.

The chemical evolution of hot WDs in the presence of diffusion and mass loss was studied by Unglaub \& Bues (2000). Using the luminosity dependence of the mass-loss rate of Blöcker (1995), they predicted that until $T_{\text {eff }}=65 \mathrm{kK}$ the $\mathrm{C}$ abundance of a $0.529 M_{\odot}$ star is reduced only by a factor of two. For stars with higher mass this occurs somewhat earlier, as indicated by the upper dashed line in Fig. 3. This figure also shows the locations of all analyzed DO WDs, PG 1159, and O(He) stars compared with VLTP evolutionary tracks from Althaus et al. (2009). As a consequence of increasing gravity and decreasing mass-loss rates, the effect of gravitational settling becomes more and more apparent. When the star has reached the lower dashed line in Fig. 3, the $\mathrm{C}$ abundance is expected to be reduced by a factor of ten. If the dependence of mass loss on the chemical composition is considered, an even sharper transition of PG 1159 stars into DO WDs is expected. We also show in Fig. 3 the corresponding theoretical wind limit for PG 1159 stars in which gravitational settling overcomes radiation-driven mass loss (thin solid line). The thick solid line in Fig. 3 corresponds to a ten times lower mass-loss rate and relates to the observed PG 1159 wind limit because no PG 1159 star is observed beyond that region. Assuming a lower mass-loss rate, however, also implies that the decrease of $\mathrm{C}$ abundances in DO WDs occurs earlier. Unglaub \& Bues (2000) claimed that the co-existence of PG 1159 stars and DO WDs with various compositions and similar stellar parameters does not contradict an evolutionary link. Depending on their mass-loss rates, some objects may evolve into DO WDs somewhat earlier, others later.

Figure 4 illustrates the observed (logarithmic) $\mathrm{C}$ abundances as a function of $T_{\text {eff }}$ for PG 1159 stars (values taken from Geier et al. in prep.; Werner \& Rauch 2014; Werner et al. 2014; Schuh et al. 2008; Werner \& Herwig 2006; Hügelmeyer et al. 2006), $\mathrm{O}(\mathrm{He})$ stars (Reindl et al. 2014; Werner et al. 2014; Wassermann et al. 2010), C-rich He-sdOs (Németh et al. 2012; Hirsch 2009), DO WDs, (this work; Werner et al. 2014; Hügelmeyer et al. 2006; Dreizler \& Werner 1996), DB WDs (Provencal et al. 1996, 2000; Dufour et al. 2002; Petitclerc et al. 2005; Desharnais et al. 2008; Koester et al. 2014) and DQ WDs (Koester \& Knist 2006; Dufour et al. 2005). Although there are some He-rich luminous PG 1159 stars, the vast majority of the luminous, that is, less evolved, PG 1159 stars (black, open triangles) display $C \approx 0.5$. Obviously, PG 1159 stars close to the wind limit $\left(T_{\text {eff }} \leq 120 \mathrm{kK}\right.$ and $\log g \geq 7.5$, black, filled triangles) display the lowest $\mathrm{C}$ abundances ( $C \leq 0.22$, by mass), which means that they are lower by about a factor of two than the average of the luminous stars. This observational fact indeed supports the theory of advancing gravitational settling. 


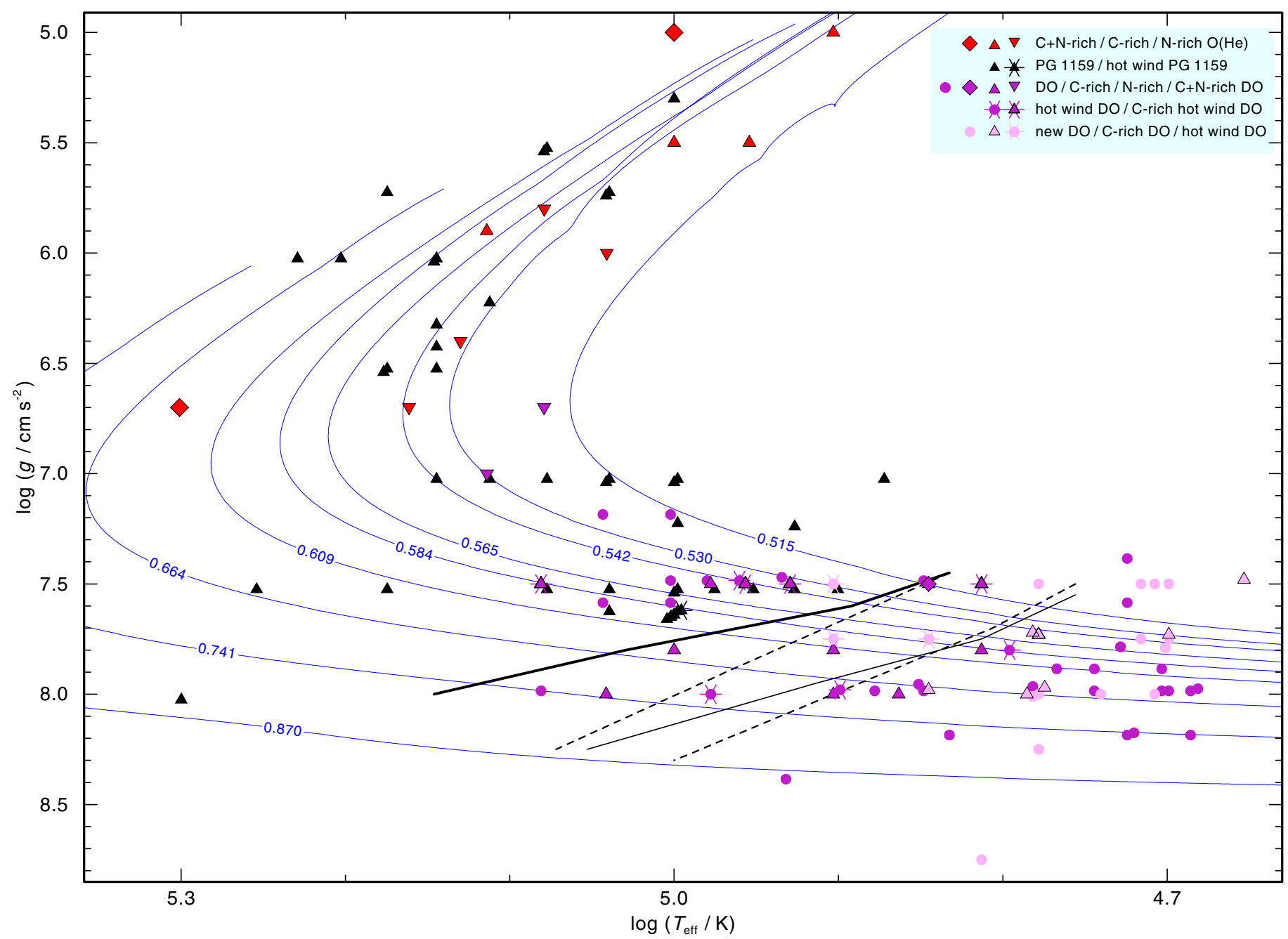

Fig. 3. Locations of O(He) stars (red, Reindl et al. 2014; Werner et al. 2014; Wassermann et al. 2010; De Marco et al. 2014), PG 1159 stars (black, Geier et al. in prep.; Werner et al. 2014; Gianninas et al. 2010; Werner \& Herwig 2006; Schuh et al. 2008) and DO WDs (purple, this work, Werner et al. 2014; Mahsereci 2011; Hügelmeyer et al. 2006; Dreizler \& Werner 1996; Nagel et al. 2006) in the $\log T_{\text {eff }}-\log g$ plane compared to VLTP post-AGB (solid lines) evolutionary tracks (labeled with stellar masses in $M_{\odot}$ ) of Althaus et al. (2009). The black solid and dashed lines indicate theoretical wind limits for abundance changes as predicted by Unglaub \& Bues (2000) and discussed in Sect. 4.2.

On the other hand, the typical $\mathrm{C}$ abundances in DO WDs (0.001-0.01 by mass) do not change along the WD cooling track and are much closer to the $\mathrm{C}$ abundances observed in $\mathrm{C}$-rich $\mathrm{O}(\mathrm{He})$ stars (typically $0.1-0.03$ by mass, Werner et al. 2014; Reindl et al. 2014; Wassermann et al. 2010) than to those of PG 1159 stars. The discovery of a significant amount of $C$ in the atmosphere of J0301, and still some detectable C in the atmosphere of J2239, emphasizes that gravitational settling might work less well than predicted by theory in the DO WD cooling region. According to Unglaub \& Bues (2000), the C abundances of J0301 and J2239 should already have dropped far below the detectable limit. Dreizler (1999) also found no evidence for gravitational settling. By analyzing HST spectra of DO WDs ranging from $T_{\text {eff }}=50-100 \mathrm{kK}$, he found that DO WDs can best be fitted with chemically homogeneous models, whereas the stratified models significantly deviated from the observations.

It is also interesting to note that the hot-wind DO WDs are located around the PG 1159-DO transition region predicted by Unglaub \& Bues (2000). Given that the cause for their spectral anomaly is a fast wind, this would additionally stress that some mass-loss must still be occurring in DO WDs.
The observed $\mathrm{C}$ abundances found in DO WDs are also very similar to those observed in C-rich He-sdO stars. A non-LTE analysis of a large sample of these stars revealed $\mathrm{C}$ abundances in the range of 0.001-0.03 (Németh et al. 2012; Hirsch 2009). Especially $\mathrm{J} 2239$, for which we derived a mass of $0.47 M_{\odot}$, might be a good candidate for a successor of a C-rich $\mathrm{He}$-sdO star.

Traces of C (0.9-9.5 $\times 10^{-6}$, by mass) are also found in some hot DB WDs (Provencal et al. 1996, 2000; Dufour et al. 2002; Petitclerc et al. 2005; Desharnais et al. 2008; Koester et al. 2014) and cannot be easily explained either by any physical processes currently thought to operate in the envelopes of DB stars. Numerical simulations of Fontaine \& Brassard (2005) showed that assuming a weak stellar wind of about $10^{-13} M_{\odot} / y r$ would sufficiently slow down the settling of $\mathrm{C}$. However, their wind model has no physical basis and is not compatible with the thin radiatively driven winds discussed by Unglaub \& Bues (2000) at much higher $T_{\text {eff }}$. Moreover, the mass-loss rates are far below the detection limit.

The monotonic decrease of the $\mathrm{C}$ abundance with decreasing temperature in DQ stars, uncovered by Dufour et al. (2005) 
Table 3. Identification of ultrahigh excitation features in J0717, J0747, and J0201.

\begin{tabular}{|c|c|c|c|c|}
\hline Ion & $\lambda / \AA$ & J0717 & J0747 & J0201 \\
\hline \multirow[t]{2}{*}{$\mathrm{CV}$} & $4945^{4}$ & $x$ & & \\
\hline & $7694^{2}$ & $\star$ & $\star$ & $\star$ \\
\hline \multirow{4}{*}{$\mathrm{C}$ VI } & $4499^{5}$ & $x$ & & \\
\hline & $5291^{5}$ & $\times$ & $\star$ & \\
\hline & $6201^{5}$ & $\times$ & $\star$ & \\
\hline & $7717^{2}$ & $\star$ & $\star$ & \\
\hline \multirow[t]{3}{*}{ N VI } & $4501^{1}$ & $\times$ & & \\
\hline & $5293^{1}$ & $\times$ & $\star$ & $\times$ \\
\hline & $6204^{1}$ & $x$ & $\star$ & $x$ \\
\hline \multirow[t]{6}{*}{ N VII } & $3887^{5}$ & $\times$ & $\star$ & $\times$ \\
\hline & $4550^{5}$ & $\times$ & $\star$ & $\times$ \\
\hline & $5669^{5}$ & $x$ & $\star$ & $\times$ \\
\hline & $6085^{5}$ & $x$ & $\star$ & $\times$ \\
\hline & $7921^{1}$ & $\star$ & $\star$ & \\
\hline & $7926^{1}$ & $\star$ & $\star$ & \\
\hline \multirow[t]{6}{*}{ O VII } & $3889^{1}$ & $x$ & $\star$ & $x$ \\
\hline & $4558^{1}$ & $x$ & $\star$ & $x$ \\
\hline & $5673^{1}$ & $\times$ & $\star$ & $x$ \\
\hline & $6088^{1}$ & $\times$ & $\star$ & $\times$ \\
\hline & $8139^{3}$ & $\star$ & & \\
\hline & $8146^{3}$ & $\star$ & & \\
\hline \multirow[t]{7}{*}{ O VIII } & $4340^{5}$ & $x$ & $\star$ & \\
\hline & $4658^{5}$ & $\times$ & $\star$ & $\times$ \\
\hline & $6064^{5}$ & $x$ & $\star$ & $\times$ \\
\hline & $6068^{5}$ & $x$ & $\star$ & $\times$ \\
\hline & $7726^{1}$ & $\star$ & $\star$ & \\
\hline & $7736^{1}$ & $\star$ & $\star$ & $\times$ \\
\hline & $8202^{1}$ & $\star$ & $\star$ & \\
\hline \multirow[t]{2}{*}{$\mathrm{Ne}$ IX } & $4797^{1}$ & $x$ & $\star$ & $\star$ \\
\hline & $6484^{1}$ & $\times$ & $\star$ & $x$ \\
\hline $\mathrm{Ne} X$ & $5252^{1}$ & $x$ & $\star$ & $x$ \\
\hline
\end{tabular}

Notes. $\times$ denotes that these lines were already identified in J0717 by Werner et al. (1995) and in J0201 by Dreizler et al. (1995), while $\star$ denotes newly identified lines. ${ }^{(1)}$ Hydrogenic value; (2) Engstrom et al. (1992); ${ }^{(3)}$ Accad et al. (1971); ${ }^{(4)}$ Moore (1970); ${ }^{(5)}$ Garcia \& Mack (1965).

and confirmed by Koester \& Knist (2006), can be explained in terms of the dredge-up model developed by Pelletier et al. (1986). They predicted that the highest contamination occurs at around $T_{\text {eff }}=12 \mathrm{kK}$, approximately the $T_{\text {eff }}$ at which the base of the He convection zone reaches its highest depth. Below this $T_{\text {eff }}$, $\mathrm{C}$ pollution decreases with further cooling, mainly because $\mathrm{C}$ sinks back into the star as a result of its partial recombination. The tight observational sequence found by Dufour et al. (2005) and Koester \& Knist (2006) allowed Brassard et al. (2007) to pin down the masses of the He-dominated envelopes in DQ stars $\left(10^{-2}\right.$ to $\left.10^{-3.75} M_{\odot}\right)$, which agrees with (V)LTP models. Hence, they reaffirmed the natural connection between PG 1159 stars, DO, DB, and DQ WDs. A completely different result was obtained by Scóccola et al. (2006). They found that PG 1159 stars cannot be related to any DQ WDs with low $\mathrm{C}$ abundances and instead suggested that the latter could be successors of RCB stars or C-poor post-extreme horizontal branch (EHB) stars. In that sense, we propose that $\mathrm{O}(\mathrm{He})$ stars should also be investigated as possible progenitors of DQ WDs. It is important to note, however, that Scóccola et al. (2006) failed to reproduce the decrease of the $\mathrm{C}$ abundance with decreasing temperature in DQ stars and instead found an increase of the $\mathrm{C}$ abundance with decreasing temperature.

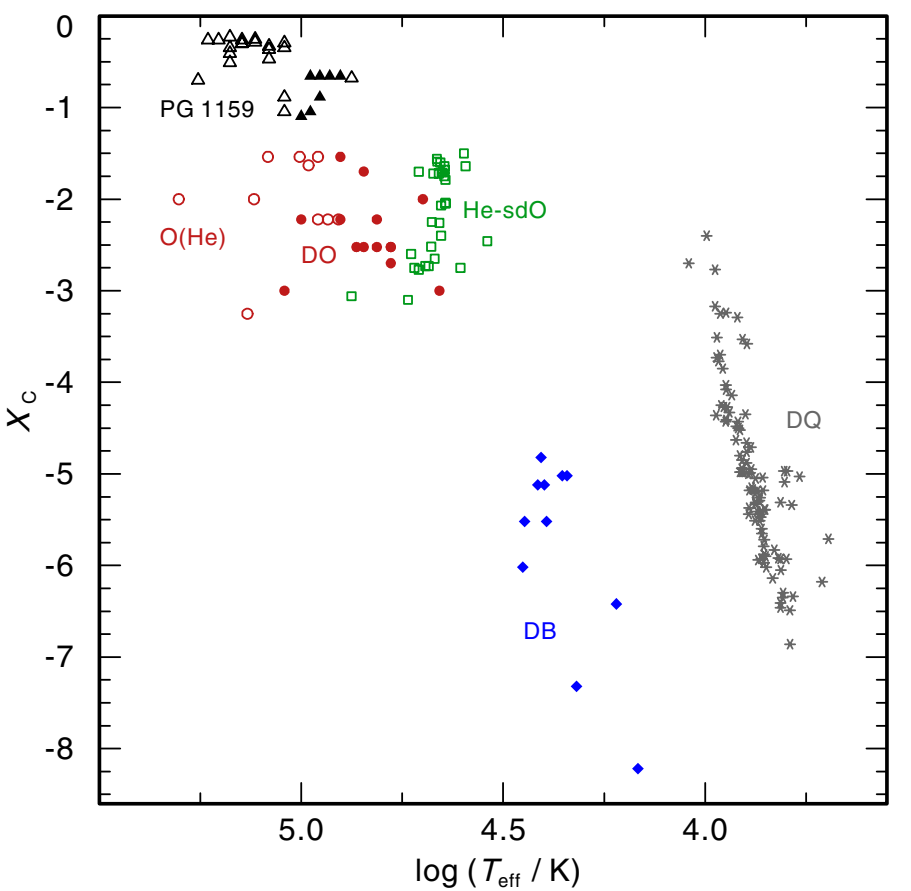

Fig. 4. Carbon abundances (in logarithmic mass fractions) before and along the non-DA WD cooling track. Luminous PG 1159 stars with $T_{\text {eff }}>120 \mathrm{kK}$ and $\log g<7.5$ are represented by open, black triangles, PG 1159 stars close to the wind limit with $T_{\text {eff }} \leq 120 \mathrm{kK}$ and $\log g \geq 7.5$ by filled, black triangles, $\mathrm{O}(\mathrm{He})$ stars and $\mathrm{DO}$ WDs before the wind limit by open, red circles, DO WDs beyond the wind limit by filled, red circles, DB WDs by blue rhombs, and DQ WDs by gray stars.

\subsection{Mass distribution}

We derived the masses of the new cool DO WDs by comparing their positions in the $\log T_{\text {eff }}-\log g$ plane with VLTP evolutionary tracks from Althaus et al. (2009, Fig. 3). With $T_{\text {eff }}=65 \mathrm{kK}$ and $\log g=8.75$, J1138 clearly lies outside of the region covered by the VLTP tracks. Considering J1138 as CO-WD, we used CO-WD evolutionary tracks of Wood (1994), which extend up to $1.10 M_{\odot}$. We found $M=1.09 M_{\odot}$ with a lower limit of $0.79 M_{\odot}$. Considering J1138 as an ONe-WD we used ONe-WD tracks of Althaus et al. (2005a) and derive $M=1.07 M_{\odot}$ with an upper limit of $1.26 M_{\odot}$. Lau et al. (2012) showed that because of instabilities in the late thermally pulsing-AGB phase of massive AGB stars with zero-age main-sequence masses of 7-10 $M_{\odot}$ and envelope masses of about $1-2 M_{\odot}$, most of the envelope of these stars can be ejected. The outcome might be a central star (CS) of around $1 M_{\odot}$ with a relatively massive planetary nebula (PN), like the so-far unique He-rich CS N66 (SMP 83), whose luminosity corresponds to a core mass of about $1.2 M_{\odot}$ (Hamann et al. 2003). J1138 could be a successor of such a star. The very low frequency of massive DO WDs could be consistent with the fact that not all AGB stars going through such an ejection mechanism would eject all of their envelope and thus would instead become a massive DA WD.

The derived masses together with their errors (as resulting from the uncertainties in $T_{\text {eff }}$ and $\log g$ ) are given in Table 2. For $\mathrm{J} 1138$, we adopted the mean value given by both sets of tracks.

In the upper panel of Fig. 5, we show the mass distribution of the DO WDs of our sample. We derived a mean mass of $\langle M\rangle=$ $0.60 M_{\odot}$ with a standard deviation of $\sigma=0.13 M_{\odot}$. In the middle panel of Fig. 5 we show the mass distribution of all analyzed PG 1159 stars, O(He) stars, and DO WDs before the observed 


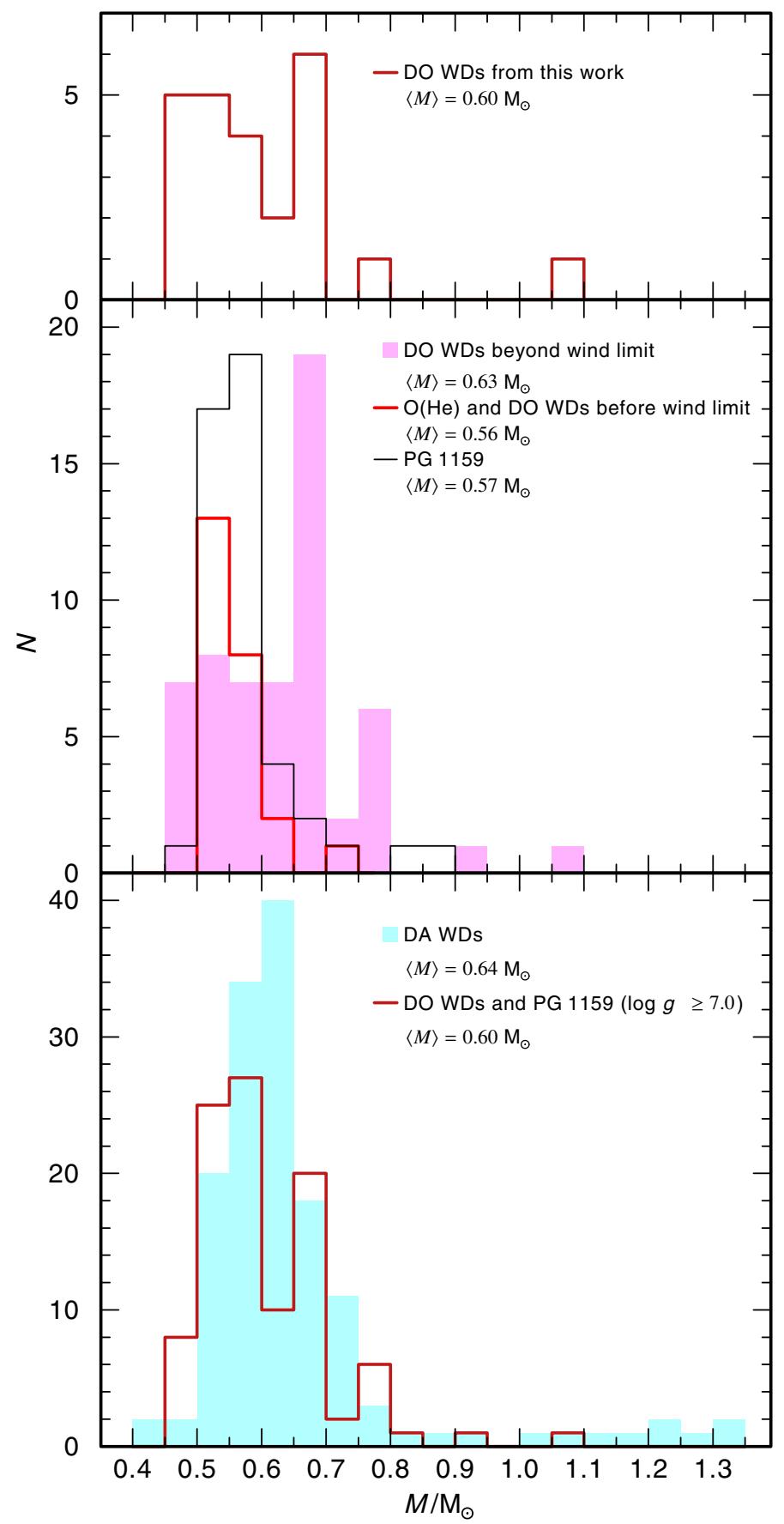

Fig. 5. Upper panel: mass distribution of all DO WDs from our sample. Middle panel: mass distribution of PG 1159 stars (thin black line), $\mathrm{O}(\mathrm{He})$ stars and DO WDs before the wind limit (thick red line), and DO WDs beyond the wind limit (gray area, light pink in the online version). Lower panel: mass distribution of the hot $\left(T_{\text {eff }} \geq 45 \mathrm{kK}\right)$ DA WDs (gray area, light blue in the online version) from the sample of Gianninas et al. (2011) compared with the mass distribution of non-DA WDs (PG 1159 and DO WDs with $\log g \geq 7$, thick red line).

wind limit ${ }^{9}$ and DO WDs beyond the wind limit. To avoid systematic errors introduced by using different evolutionary tracks, we derived the masses for PG 1159 and $\mathrm{O}(\mathrm{He})$ stars by additionally using the VLTP tracks from Althaus et al. (2009). The mean mass of the $\mathrm{O}(\mathrm{He})$ stars and DO WDs before the wind limit

\footnotetext{
9 Since no gravitational settling is predicted for DO WDs before the wind limit, we placed them in the $\mathrm{O}(\mathrm{He})$ group.
}

$\left(\langle M\rangle=0.56 M_{\odot}\right.$, with $\left.\sigma=0.04 M_{\odot}\right)$ is slightly lower than the mean mass of the PG $1159 \operatorname{stars}\left(\langle M\rangle=0.57 M_{\odot}, \sigma=0.08 M_{\odot}\right)$. The mean mass of DO WDs beyond the wind limit is slightly higher $\left(\langle M\rangle=0.63 M_{\odot}, \sigma=0.11 M_{\odot}\right)$ than those of the objects before the wind limit. This trend continues in the mass distribution of DB WDs. Using only the spectra with $S / N \geq 15$ out of their sample of 923 DB stars, Kleinman et al. (2013) found a mean mass of $\langle M\rangle=0.685 \pm 0.013 M_{\odot}$. By restricting this sample to just those hotter than $T_{\text {eff }}=16 \mathrm{kK}$, they found a slightly lower mean mass of $\langle M\rangle=0.676 \pm 0.014 M_{\odot}$. Bergeron et al. (2011) presented a detailed analysis of 108 DB WDs based on model atmosphere fits to high $\mathrm{S} / \mathrm{N}$ optical spectroscopy. They derived an almost identical mean mass of $0.67 M_{\odot}$ for their sample.

The phenomenon of increasing mean mass with decreasing $T_{\text {eff }}$ is not restricted to non-DA WDs. Gianninas et al. (2011) presented the spectroscopic analysis of over 1100 bright $(V \geq 17.5)$ DA white dwarfs. They found a mean mass of $\langle M\rangle=0.661 M_{\odot}$, with $\sigma=0.160 M_{\odot}$, but by dividing their sample into objects with $T_{\text {eff }}>13 \mathrm{kK}$ and $T_{\text {eff }}<13 \mathrm{kK}$, they also found that the mean mass of the hot objects $\left(\langle M\rangle=0.638 M_{\odot}, \sigma=0.145 M_{\odot}\right)$ is significantly lower than the mean mass of the cool DA WDs $\left(\langle M\rangle=0.736 M_{\odot}, \sigma=0.183 M_{\odot}\right)$. Tremblay et al. (2013) showed that this problem can be solved using 3D hydrodynamical models to compute spectra for the cool DA WDs. In the lower panel of Fig. 5 we therefore compare only the mass distributions of DA WDs with $T_{\text {eff }}>45 \mathrm{kK}$ with those of the DO WDs and PG 1159 stars with $\log g \geq 7.0$. We derived mean masses of both classes to $\langle M\rangle=0.64 M_{\odot}$ (hot DA WDs) and $\langle M\rangle=0.60 M_{\odot}$ (non-DA WDs). The slightly higher mean mass for DA WDs results from the larger number of massive WDs, while a larger number of non-DA WDs with $M<0.5 M_{\odot}$ is known. Seven out of the 55 (about 13\%) DO WDs beyond the wind limit have $M<0.5 M_{\odot}$, thus they are most likely post-EHB stars. The progenitors of these DO WDs might be He-sdO stars or low mass PG 1159 stars, such as HS 0704+6153 (Dreizler \& Heber 1998). However, we emphasize that the errors on the masses are still large and that only a better $\log g$ determination can provide better constraints.

Another interesting feature that can be seen in the middle panel of Fig. 5 is that the mass distribution of the DO WDs beyond the wind limit compared with the mass distribution of the objects before the wind limit strongly disagree. While the flat plateau around $0.6 M_{\odot}$ in the mass distribution of the DO WDs beyond the wind limit agrees with the mass distribution of PG 1159 stars, $\mathrm{O}(\mathrm{He})$ stars, and DO WDs before the wind limit, two additional peaks at $0.675 M_{\odot}$ and $0.775 M_{\odot}$ can be seen. The significant difference in the mass distributions is confirmed by a Kolmogorov-Smirnov test. The result of this test is that the probability that both samples are taken from the same mass distribution is rather small $\left(<10^{-4}\right)$. The shape of the mass distributions before and beyond the wind limit are also preserved if we divide the stars into objects younger and older than 0.4 Myr, which approximately corresponds to a vertical line at $T_{\text {eff }}=80 \mathrm{kK}$ (Althaus et al. 2009). This shows that the discrepancy in the mass distributions does not arise because the wind limit is reached earlier for more massive stars. Althaus et al. (2009) noted that the mass distribution of young DO WDs differs considerably from that of the older WDs. Werner et al. (2014) attributed the higher mean mass for DOs found by Hügelmeyer et al. (2006) to a calibration problem in the SDSS DR4. However, we found that using only the DO WDs from our analysis, we also see a peak around $0.675 M_{\odot}$. Thus 
we believe that this peak is real and not an artifact of a poor flux calibration.

This poses the question whether these two high-mass peaks display different input channels of DO WDs. Althaus et al. (2009) suggested that some DO WDs might result from evolutionary channels that do not involve PG 1159 stars. For instance, they could be the result of post-merger evolution involving $\mathrm{RCB}$, EHe, luminous He-sdO stars, and $\mathrm{O}(\mathrm{He})$ stars. Reindl et al. (2014) found that using double He-WD merger tracks from Zhang \& Jeffery (2012b) instead of VLTP tracks, the masses of $\mathrm{O}(\mathrm{He})$ stars are $0.07-0.16 M_{\odot}$ higher. This would indeed shift the mean mass of these stars closer to the higher mass peaks of DO WDs beyond the wind limit. Comparing the evolutionary tracks of Zhang \& Jeffery (2012b) and Althaus et al. (2009), we found that they differ significantly only in the luminous part $\left(T_{\text {eff }}>100 \mathrm{kK}\right)$ in the $\log T_{\text {eff }}-\log g$ plane. We again derived the masses of all $\mathrm{O}(\mathrm{He})$ stars and DO WDs before the wind limit using the tracks of Zhang \& Jeffery (2012b) and found a mean mass of $\langle M\rangle=0.62 M_{\odot}$ instead of the $\langle M\rangle=0.56 M_{\odot}$ found using the tracks of Althaus et al. (2009). Although a double He-WD merger can be excluded for some $\mathrm{O}(\mathrm{He})$ stars, we claim that a contribution of post-double He-WD mergers to the observed higher mass peaks in the mass distribution of the DO WDs beyond the wind limit is possible. We also stress that determining the mass through evolutionary tracks requires knowing the evolutionary history of an object, which is very unclear at least for the He-dominated objects.

Another possible origin of DO WDs with higher masses are H-deficient [WC] or [WN]-type central stars. A spectroscopic mass determination for these objects is, however, extremely difficult since $\log g$ cannot be derived from photospheric lines. Wind emission lines do not depend on the first approximation of $\mathrm{L}$ and M. For the spectral analysis of these stars, it is common practice to assume standard values of $\log g$ or $M$ (e.g., $\log g=6.0$ or $\left.M=0.6 M_{\odot}\right)$, which are equivalent input parameters for wind codes. The two currently known [WN]-type central stars IC 4663 (Miszalski et al. 2012) and Abell 48 (Todt et al. 2013; Frew et al. 2014) are in an evolutionary state similar to or even later than the $\mathrm{O}(\mathrm{He})$ stars, but show much stronger stellar winds. Reindl et al. (2014) speculated that [WN] stars are $\mathrm{O}(\mathrm{He})$ stars, but with higher masses and hence higher luminosity, which could explain the higher mass-loss rates of [WN] stars.

\subsection{Ratio of DAs to non-DAs for hot WDs}

The sample of Gianninas et al. (2011) comprised 131 DA WDs with $T_{\text {eff }}>45 \mathrm{kK}$, while there are 49 PG 1159 stars with $\log g \geq$ 7.0, and DO WDs (hot non-DA WDs) known with $V \leq 17.5$. This would lead to a ratio of hot DAs to non-DAs of 2.7. However, neither sample can be considered complete. To correctly calculate the ratio of hot DAs to non-DAs, it is necessary to compare samples with the same the magnitude limit, sky coverage, and completeness. No systematic search for hot DA WDs in the SDSS DR 10 was presented so far, therefore we restricted ourselves to the SDSS DR7 spectroscopic sample. Since we compare objects in the same $T_{\text {eff }}$ range, incompleteness should affect both subclasses in the same manner. Atmospheric parameters derived by Kleinman et al. (2013) are only based on LTE models, and thus they show large differences for $T_{\text {eff }} \gtrsim 50 \mathrm{kK}$ compared with the objects they had in common with the sample of Gianninas et al. (2011). However, to distinguish whether a DA WD is hotter or cooler than $T_{\text {eff }}=45 \mathrm{kK}$, LTE models are probably good enough. By comparing pure $\mathrm{He}$ and $\mathrm{H}$ model atmosphere fluxes, we found that at $T_{\text {eff }}=45 \mathrm{kK}$ DA WDs are about 0.1 mag brighter than DO WDs. Accordingly, we set the magnitude limit for DA WDs to $g<17.4$ and for DO WDs to $g<17.5$. We found in total 117 DA WDs with $T_{\text {eff }}>45 \mathrm{kK}$, with 81 of them clean DA WDs (no subtypes, e.g. DAO, DAM, DAH). On the other hand we found 23 hot non-DA WDs in the SDSS DR7 spectroscopic sample with $g<17.5$, leading to $\mathrm{DA} /$ non-DA $=5.1$, with a lower limit of 3.5 if we consider clean DA WDs alone.

Among the $\mathrm{H}$-deficient objects before the wind limit that are included in the SDSS DR 10 spectroscopic sample, we find 18 PG 1159 stars and $12 \mathrm{O}(\mathrm{He})$ stars and DO WDs, which leads to a ratio of $\mathrm{C}$-dominated to $\mathrm{He}$-dominated objects of 1.5 . Including $\log g>7.0$ objects alone, the ratio of C-dominated to $\mathrm{He}$ dominated objects is 0.75 . This suggests that DO WDs beyond the wind limit may be fed to a similar extent by PG 1159 and $\mathrm{O}(\mathrm{He})$ stars.

\subsection{Conclusions}

We have visually scanned a color-selected sample of white dwarf candidates in the SDSS DR10 and identified 22 new cool DO white dwarfs. Effective temperatures, surface gravities, and C abundances (or at least upper limits) for 24 DO WDs were derived with non-LTE model atmospheres. Among the newly identified DO WDs, we found one more member of the so-called hot-wind DO WDs, which shows uhei absorption lines. One of the DO WDs is the most massive DO WD ever discovered with a mass of $1.07 M_{\odot}$ if it is an ONe-WD or $1.09 M_{\odot}$ if it is a CO-WD. Two of our objects are the coolest DO WDs ever discovered that still show a considerable amount of $\mathrm{C}$ in the atmosphere $(C=0.001-0.01)$. This strongly contradicts the diffusion calculations of Unglaub \& Bues (2000). We suggest that - similar to what has been proposed for the cooler DB stars - a weak mass-loss is present in cool DO WDs. Furthermore, we presented the mass distribution of all hitherto analyzed DO WDs, PG 1159, and $\mathrm{O}(\mathrm{He})$ stars. We found that the mass distribution of DO WDs beyond the wind limit strongly deviates from the mass distribution of the objects before the wind limit and explained this phenomenon with a scenario of different input channels. The plateau around $0.6 M_{\odot}$ in the mass distribution of DO WDs beyond the wind limit agrees with the mass distribution of PG 1159 stars, $\mathrm{O}(\mathrm{He})$ stars, and DO WDs before the wind limit. The two additional higher mass peaks might reflect a merger origin of some $\mathrm{O}(\mathrm{He})$ stars and DO WDs and/or the possibility that [WN] and [WC] type central stars are more massive than PG1159 and $\mathrm{O}(\mathrm{He})$ stars. The non-DA WD channel may be fed by about 13\% by low-mass PG 1159 stars and post-EHB He-sdO stars. PG 1159 stars and $\mathrm{O}(\mathrm{He})$ stars may contribute to a similar extent to the non-DA WD channel.

Acknowledgements. N.R. is supported by the German Research Foundation (DFG, grant WE 1312/41-1), T.R. by the German Aerospace Center (DLR, grant $05 \mathrm{OR}$ 1401). The research leading to these results has received funding from the European Research Council under the European Union's Seventh Framework Programme (FP/2007-2013)/ERC Grant Agreement No. 320964 (WDTracer). B.T.G. was supported in part by the UK's Science and Technology Facilities Council (ST/I001719/1). Funding for SDSS-III has been provided by the Alfred P. Sloan Foundation, the Participating Institutions, the National Science Foundation, and the US Department of Energy Office of Science. The SDSS-III web site is http://www.sdss3.org/. SDSS-III is managed by the Astrophysical Research Consortium for the Participating Institutions of the SDSS-III Collaboration including the University of Arizona, the Brazilian Participation Group, Brookhaven National Laboratory, Carnegie Mellon University, University of Florida, the French Participation Group, the German Participation Group, Harvard University, the Instituto de Astrofisica de Canarias, the Michigan State/Notre Dame/JINA Participation Group, Johns 
Hopkins University, Lawrence Berkeley National Laboratory, Max Planck Institute for Astrophysics, Max Planck Institute for Extraterrestrial Physics, New Mexico State University, New York University, Ohio State University, Pennsylvania State University, University of Portsmouth, Princeton University, the Spanish Participation Group, University of Tokyo, University of Utah, Vanderbilt University, University of Virginia, University of Washington, and Yale University. This research has made use of the SIMBAD database, operated at CDS, Strasbourg, France. This research has made use of the VizieR catalogue access tool, CDS, Strasbourg, France.

\section{References}

Accad, Y., Pekeris, C. L., \& Schiff, B. 1971, Phys. Rev. A, 4, 516 Ahn, C. P., Alexandroff, R., Allende Prieto, C., et al. 2012, ApJS, 203, 21 Althaus, L. G., García-Berro, E., Isern, J., \& Córsico, A. H. 2005a, A\&A, 441, 689

Althaus, L. G., Serenelli, A. M., Panei, J. A., et al. 2005b, A\&A, 435, 631 Althaus, L. G., Panei, J. A., Miller Bertolami, M. M., et al. 2009, ApJ, 704, 1605 Asplund, M., Grevesse, N., Sauval, A. J., \& Scott, P. 2009, ARA\&A, 47, 481 Barnard, A. J., Cooper, J., \& Shamey, L. J. 1969, A\&A, 1, 28

Barnard, A. J., Cooper, J., \& Smith, E. W. 1974, J. Quant. Spectr. Rad. Transf., 14,1025

Bergeron, P., Wesemael, F., Dufour, P., et al. 2011, ApJ, 737, 28

Blöcker, T. 1995, A\&A, 299, 755

Brassard, P., Fontaine, G., Dufour, P., \& Bergeron, P. 2007, in 15th European Workshop on White Dwarfs, eds. R. Napiwotzki, \& M. R. Burleigh, ASP Conf. Ser., 372, 19

De Marco, O., Long, J., George, H. J., et al. 2014, MNRAS, submitted Desharnais, S., Wesemael, F., Chayer, P., Kruk, J. W., \& Saffer, R. A. 2008, ApJ, 672,540

Dreizler, S. 1999, A\&A, 352, 632

Dreizler, S., \& Heber, U. 1998, A\&A, 334, 618

Dreizler, S., \& Werner, K. 1996, A\&A, 314, 217

Dreizler, S., Heber, U., Napiwotzki, R., \& Hagen, H. J. 1995, A\&A, 303, L53

Dufour, P., Wesemael, F., \& Bergeron, P. 2002, ApJ, 575, 1025

Dufour, P., Bergeron, P., \& Fontaine, G. 2005, ApJ, 627, 404

Dufour, P., Liebert, J., Fontaine, G., \& Behara, N. 2007, Nature, 450, 522

Eisenstein, D. J., Liebert, J., Harris, H. C., et al. 2006, ApJS, 167, 40

Engstrom, L., Bengtsson, P., Jupen, C., \& Westerlind, M. 1992, J. Phys. B At. Mol. Phys., 25, 2459

Fontaine, G., \& Brassard, P. 2005, in 14th European Workshop on White Dwarfs, eds. D. Koester, \& S. Moehler, ASP Conf. Ser., 334, 49

Frew, D. J., Bojičić, I. S., Parker, Q. A., et al. 2014, MNRAS, 440, 1345

Gänsicke, B. T., Koester, D., Girven, J., Marsh, T. R., \& Steeghs, D. 2010, Science, 327,188

Gianninas, A., Bergeron, P., Dupuis, J., \& Ruiz, M. T. 2010, ApJ, 720, 581

Gianninas, A., Bergeron, P., \& Ruiz, M. T. 2011, ApJ, 743, 138

Griem, H. R. 1974, Spectral line broadening by plasmas (New York: Academic Press), 39, 421

Hamann, W.-R., Peña, M., Gräfener, G., \& Ruiz, M. T. 2003, A\&A, 409, 969

Heber, U., Dreizler, S., \& Hagen, H.-J. 1996, A\&A, 311, L17

Hirsch, H. A. 2009, Ph.D. Thesis, University Nuremberg, Germany

Hügelmeyer, S. D., Dreizler, S., Werner, K., et al. 2005, A\&A, 442, 309

Hügelmeyer, S. D., Dreizler, S., Homeier, D., et al. 2006, A\&A, 454, 617

Iben, Jr., I., Kaler, J. B., Truran, J. W., \& Renzini, A. 1983, ApJ, 264, 605
Kleinman, S. J., Kepler, S. O., Koester, D., et al. 2013, ApJS, 204, 5

Koester, D., \& Knist, S. 2006, A\&A, 454, 951

Koester, D., Provencal, J., \& Gänsicke, B. T. 2014, A\&A, 568, A118

Lau, H. H. B., Gil-Pons, P., Doherty, C., \& Lattanzio, J. 2012, A\&A, 542, A1

Mahsereci, M. 2011, Diploma thesis, University Tübingen, Germany

Miller Bertolami, M. M. 2014, A\&A, 562, A123

Miller Bertolami, M. M., Melendez, B. E., Althaus, L. G., \& Isern, J. 2014, JCAP, 10, 069

Miszalski, B., Crowther, P. A., De Marco, O., et al. 2012, MNRAS, 423, 934

Moore, C. E. 1970, Selected tables of atomic spectra (NSRDS-NBS)

Müller-Ringat, E. 2013, Dissertation, University of Tübingen, Germany, http://tobias-lib.uni-tuebingen.de/voll texte/2013/6774/

Nagel, T., Schuh, S., Kusterer, D.-J., et al. 2006, A\&A, 448, L25

Napiwotzki, R., \& Schönberner, D. 1995, A\&A, 301, 545

Németh, P., Kawka, A., \& Vennes, S. 2012, MNRAS, 427, 2180

Nousek, J. A., Shipman, H. L., Holberg, J. B., et al. 1986, ApJ, 309, 230

Pelletier, C., Fontaine, G., Wesemael, F., Michaud, G., \& Wegner, G. 1986, ApJ, 307, 242

Petitclerc, N., Wesemael, F., Kruk, J. W., Chayer, P., \& Billères, M. 2005, ApJ, 624,317

Provencal, J. L., Shipman, H. L., Thejll, P., Vennes, S., \& Bradley, P. A. 1996, ApJ, 466, 1011

Provencal, J. L., Shipman, H. L., Thejll, P., \& Vennes, S. 2000, ApJ, 542, 1041

Rauch, T., \& Deetjen, J. L. 2003, in Stellar Atmosphere Modeling, eds. I. Hubeny, D. Mihalas, \& K. Werner, ASP Conf. Ser., 288, 103

Reindl, N., Rauch, T., Werner, K., Kruk, J. W., \& Todt, H. 2014, A\&A, 566, A116

Schöning, T. 1993, A\&A, 267, 300

Schöning, T., \& Butler, K. 1989, A\&AS, 78, 51

Schuh, S., Traulsen, I., Nagel, T., et al. 2008, Astron. Nachr., 329, 376

Scóccola, C. G., Althaus, L. G., Serenelli, A. M., Rohrmann, R. D., \& Córsico, A. H. 2006, A\&A, 451, 147

Sinamyan, P. K. 2011, Astrophysics, 54, 413

Sion, E. M. 2011, in White Dwarf Atmospheres and Circumstellar Environments (Wiley-VCH), 1

Todt, H., Kniazev, A. Y., Gvaramadze, V. V., et al. 2013, MNRAS, 430, 2302

Tremblay, P.-E., Ludwig, H.-G., Steffen, M., \& Freytag, B. 2013, A\&A, 559, A104

Unglaub, K., \& Bues, I. 2000, A\&A, 359, 1042

Wassermann, D., Werner, K., Rauch, T., \& Kruk, J. W. 2010, A\&A, 524, A9

Werner, K. 1991, A\&A, 251, 147

Werner, K., \& Herwig, F. 2006, PASP, 118, 183

Werner, K., \& Rauch. 2014, A\&A, 569, A99

Werner, K., Dreizler, S., Heber, U., et al. 1995, A\&A, 293, L75

Werner, K., Deetjen, J. L., Dreizler, S., et al. 2003, in Stellar Atmosphere Modeling, eds. I. Hubeny, D. Mihalas, \& K. Werner, ASP Conf. Ser., 288, 31

Werner, K., Rauch, T., Barstow, M. A., \& Kruk, J. W. 2004a, A\&A, 421, 1169

Werner, K., Rauch, T., Napiwotzki, R., et al. 2004b, A\&A, 424, 657

Werner, K., Rauch, T., \& Kepler, S. O. 2014, A\&A, 564, A53

Wood, M. A. 1994, in Am. Astron. Soc. Meet. Abstr., BAAS, 26, 1382

Zhang, X., \& Jeffery, C. S. 2012a, MNRAS, 426, L81

Zhang, X., \& Jeffery, C. S. 2012b, MNRAS, 419, 452 